\title{
Evaluation Index System Construction for Emergency Management Capacity of Campus Emergencies Based on OODA Ring*
}

\author{
Yang Liu \\ Students Affairs Office \\ Wuhan University of Science and Technology \\ Wuhan, China 430081 \\ Shangwen Peng \\ School of Machinery and Automation \\ Wuhan University of Science and Technology \\ Wuhan, China 430081 \\ Chen Zhang \\ School of Machinery and Automation \\ Wuhan University of Science and Technology \\ Wuhan, China 430081
}

\author{
Yufei Li \\ College of Resources and Environmental Engineering, \\ Wuhan University of Science and Technology \\ Wuhan, China 430081 \\ Fang Fang \\ School of Machinery and Automation \\ Wuhan University of Science and Technology \\ Wuhan, China 430081 \\ Hao Qian \\ School of Machinery and Automation \\ Wuhan University of Science and Technology \\ Wuhan, China 430081
}

\begin{abstract}
Through the analysis of the concept, definition and classification of emergency management in colleges and universities, combing the research dimension of campus emergency management, clarifying the characteristics of emergency management in campus emergencies, introducing OODA ring theory and establishing a new mode of emergency management for campus emergencies. The principles of scientificity, qualitative and quantitative integration, availability and dynamics are selected, and its evaluation index system is built based on the evaluation index of emergency management capacities.
\end{abstract}

Keywords-campus emergencies; emergency management; $O O D A$ ring theory; evaluation index system

\section{INTRODUCTION}

The emergencies have the following typical characteristics: they are unpredictable before they occur, they are sudden when they occur, they may cause or have caused serious harm to society after they occur, and they are harmful to the safety of public order. In the process of managing emergencies, it is a very important but complicated task to establish a scientific and effective evaluation index system. Its working principle is a systematic process based on effective detection and screening key factors in the early stage of an emergency, analyzing reliable survey data, ranking and sorting it reasonably. It is the basis for prevention, response, and rescue of various emergencies, and is also an important basis for

*Fund Project: Humanities and Social Sciences Research Project of Hubei Provincial Department of Education (16Y019). decision-making on emergency management programs [1].

The evaluation index system construction for emergency management capacity of campus emergencies is the premise and basis of evaluation. Whether the evaluation index system is constructed properly will directly affect evaluation results, accuracy and reliability [2]. At present, there are many researches on the evaluation of emergency management capacities in colleges and universities. There are researches from the comprehensive emergency management process [3], the crisis life cycle [4], the Hall three-dimensional structure [5], and the safety behavior [6], and also the use of the analytic hierarchy process [7], the gray correlation degree [8], BP neural network [9] and other mathematical methods for modeling research. The OODA (Observe-orient-decide-act) ring theory [10] is divided into four steps of observation, orientation, decision and action, and has a good dynamic adjustment advantage. The emergency management process in colleges and universities is typical dynamic closed-loop process, so the introduction of OODA ring theory into the emergency management of campus emergencies and the construction of corresponding evaluation index system has good theoretical and practical research significance.

\section{CONCEPT, DEFINITION AND CLASSIFICATION OF EMERGENCY MANAGEMENT CAPACITIES IN COLLEGES AND UNIVERSITIES}

\section{A. Definition of Campus Emergencies}

Colleges and universities are important places for college students to work, study and live, while college students have 
distinct characteristics, high degree of personalization, sensitive thinking and self-expression. The emergencies can easily lead to large-scale disorder and even panic and riots on campus, such as outbreaks of infectious diseases; natural disasters like typhoons and earthquakes; large-scale fires caused by students in the bedroom using illegal appliances, casualties and other vicious incidents by students gathering in groups to fight; suicide because of failure to withstand the pressure of academic, gender relations and family conflicts and so on; demonstrations by students in conflict with the colleges and universities affected by important events at home and abroad in the case of unfavorable school guidance, etc. Therefore, campus emergencies can be defined as events that cause harm to the situation caused by the daily teaching, life, and work order of the colleges and universities under the unfavorable guidance of human, social or political reasons [11].

\section{B. Definition of Emergency Management Capacity in Colleges and Universities}

Emergency management is a series of measures taken against campus emergencies, and campus emergencies are aimed at the crisis events in colleges and universities. The difference between the two situations is obvious, and the two situations exist in colleges and universities. The main and important components of campus crisis management are emergencies, which are included in the crisis of colleges and universities. In order to effectively prevent and respond to emergencies for the work, study and life of teachers and students in colleges and universities having a stable and safe guarantee, and promote the benign development of colleges and universities, so the various emergency treatment methods are called campus emergency management.

\section{Classification of Emergency Management Capacities in Colleges and Universities}

The emergency management capacities in colleges and universities can be divided into the following three categories: prevention capacity before the emergency event, situation control capacity at the time of emergency event occurring, and organizational recovery capacity after the event. The prevention capacity before the emergency event means that the colleges and universities should train emergency response units, pre-plan the emergency treatment plan, carry out relevant emergency drills such as fire escape, earthquake escape, air defense drills, etc. on campus regularly, increase the research and development of the emergency management capacity evaluation system, and increase investment in improving emergency management related facilities for possible emergency events. The situation control capacity at the time of emergency event occurring means after the evaluation of the relevant data of the emergency event, the reasonable classification according to the evaluation system is made, the corresponding plan is started according to different degrees, the field personnel are effectively organized, and the accurate information grasped is reasonably analyzed, evaluated and released in time. The organizational recovery capacity after the event means after the end of the emergency, the life and teaching order are quickly adjusted, the infrastructure is repaired, the loss is evaluated in time, the accountability is conducted, the rewards and punishments are made, the psychological counseling is arranged, all kinds of data and materials in the event are collected and archived in a timely manner, the statistics and analysis are made, and the summary report is comprehensively summarized and generated.

\section{RESEARCH DIMENSIONS OF EMERGENCY MANAGEMENT CAPACITY EVALUATION IN COLLEGES AND UNIVERSITIES}

\section{A. Comprehensive Emergency Management}

In the whole process of public emergencies, comprehensive emergency management is an emergency response, which includes a series of contents including the plan process, emergency response, recovery and reconstruction, feedback and improvement. The proposed program focuses on implementing various types of disasters and their consequences, coordinating and using all resources and configuring emergency management personnel to work.

\section{B. The Life Cycle of the Crisis}

For investigating the time of the crisis, a foreign study summarized the life cycle of the crisis and divided the life cycle according to this basis. Countries generally recognize three of them. A. Finke's four-stage life cycle model. In this theory, Finke divides the crisis into a symptom period, an attack period, a continuation period, and a recovery period. During the symptom period, some specific performances indicate that the event is in its infancy; the attack period indicates that after a period of time, the event has been fermented into a crisis; during the continuation period, the situation develops further and the crisis expands; the recovery period indicates that the crisis has been safely passed. B. Three-stage model. The three-stage model divides the crisis into three stages: early, middle and late. C. Mitroff's five-stage model. The theory divides the crisis into five stages, namely the signal detection stage, the detection and prevention stage, the damage control stage, the recovery stage, and the learning stage. The corresponding meaning of each stage is investigating and collecting relevant information that needs to be done in the early stages of the crisis, preventing and eliminating existing hazards based on hazard signals, controlling the hazard as much as possible, recovering as soon as possible afterwards, and summarizing the experience respectively.

\section{Hall Three-dimensional Structure}

Hall system engineering is another name for Hall's threedimensional structure. When faced with the planning, organization and management of large complex systems, Hall system engineering can provide a unified solution and is widely recognized. As shown in "Fig. 1", an index system is constructed. 


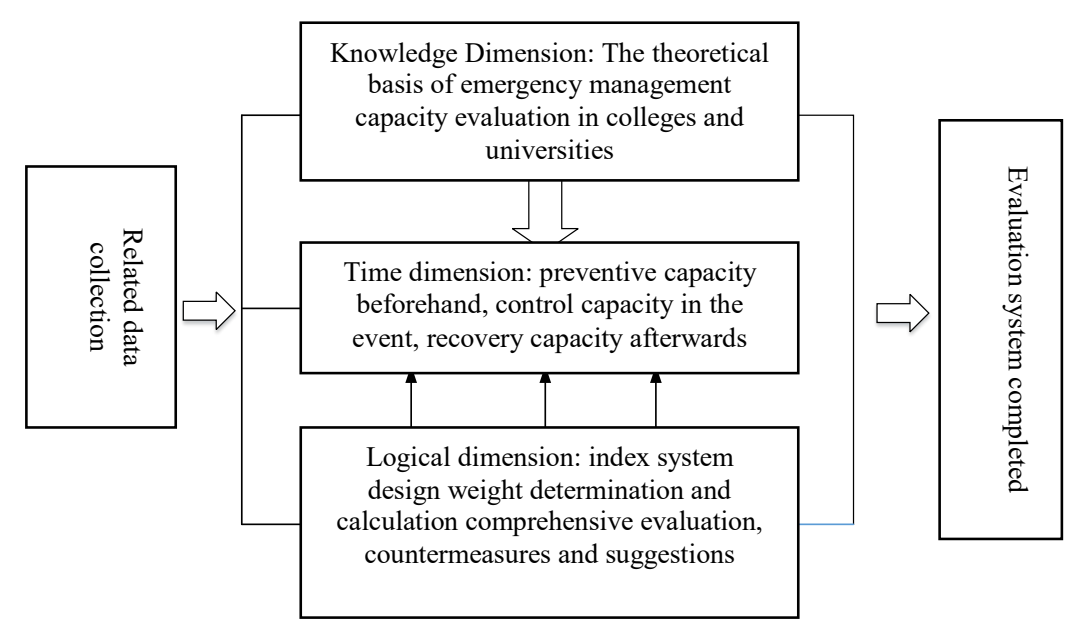

Fig. 1. Indicator System Logic Diagram.

Among them, each time node in the emergency process is called time dimension, and the relevant theoretical knowledge and methods used by colleges and universities to deal with emergencies are called knowledge dimension, and the logical dimension includes the logical thinking process of constructing index system such as determination of weights and solutions and recommendations.

\section{Theory of Safe Behavior}

Analyzing and studying the theory of certain factors affecting human behavioral safety is the theory of safe behavior. The law of safe behavior is the main part of the theory. The discipline aims to prevent unsafe and inappropriate behaviors. The safety needs to act on behavior effect for the safety awareness, certain psychological factors, and differences in individual safety behaviors in labor is the main research parts of the theory. In the process of emergency management of campus emergencies, it mainly involves the use of safe behavior theory to intervene at the psychological level. The improvement of campus emergency response capacity should be conducted by using the theory of safe behavior.

\section{PRINCIPLES For EVALUATION INDEX SySTEM CONSTRUCTION OF CAMPUS EMERGENCY MANAGEMENT CAPACITY}

In order to make a more effective and objective evaluation of emergency management capacities, and make it related to the objectivity of the evaluation results and the scientificity of the evaluation process, an indispensable part is the establishment of a campus emergency management capacity evaluation index system, which is an essential part. In order to avoid negative and negative reactions, the structure of the system and the selection of index must be scientific and reasonable; otherwise it may cause negative reactions. Therefore, the following four principles should be used as the basis for the construction of the campus emergency management capacity index system [12].

\section{A. Scientific Principle}

The principle of scientificity is the most basic principle for the construction of an index system. If the index lacks scientificity, it will lead to slight or serious deviation of the evaluation results. The scientific principle is mainly reflected in the rationality of the index system structure, the scientific selection of index, and the comprehensive coverage of index. Therefore, it can accurately reflect the actual situation of campus emergency management capacities. As the basis of the evaluation, it must include the true and reliable statistical data used in the selection of index and the objective existence. The establishment of the index system structure must conform to the scientific and objective foundation. The construction of the system must have strict logic and factual basis. In the index system, each index should also reflect the state of emergency management capacities of colleges and universities as much as possible, and comprehensively reflect the level of emergency management capacities of colleges and universities.

\section{B. Principle of Combining Qualitative and Quantitative}

All indexes can be quantified as the best embodiment of every index in the index system. However, in fact, most of the index systems are reflected in the objective quantitative indexes with subjective indexes, which inevitably contain a large number of non-figurative factors and non-quantitative components. Therefore, when the evaluation index system is carried out, the non-representational factors can only be described by subjective evaluation or qualitatively. For the quantifiable indexes, objective statistical data should be used to reflect.

\section{Principle of Availability}

The evaluation of campus emergency management capacities involves many indexes. However, incorporating all factors into the evaluation index system wastes a lot of human and material resources, and it cannot be fully realized. Some of the content is too subjective and non-conformative, so it does not have the value of being included in the evaluation system; some of the impacts on the overall level of emergency response capacity of colleges and universities can be included 
in the small factors that can't be counted. However, if considering this factor to all, it will need to waste a lot of resources but we will not get the corresponding results. Therefore, we should select representative indicators through analysis and comparison, and the evaluation index should be easy to obtain, easy to understand and convenient to quantify.

\section{Dynamic Principle}

Emergency management capacities will continue to adjust to the changes in the internal and external environment of colleges and universities as well as the society. It is not a static and balanced system. Therefore, dynamic adaptability should also be a major principle established by the campus emergency management capacity index system. So this requires the construction of the evaluation index system to take into account the changes in the colleges, universities and social environment within a certain time range that can be expected, and always keep the objective facts as the basis to establish index system.

\section{EVALUATION INDEX SYSTEM OF CAMPUS EMERGENCY MANAGEMENT CAPACITY BASED ON OODA RING}

\section{A. Ooda Ring Theory}

The OODA ring [13] process continues to perform in this complex and dynamic environment by continuously cycling and updating the four stages of observation- orientationdecision-action. In the process, the information of the decisionmaking and execution stages needs to be timely and effectively fed back to the system observation stage, so as to cope with the real-time and variability of various factors in the external environment, and then turn on the OODA cycle process again, thus achieving the transformation from "information advantage" to "action advantage". The OODA ring process is shown in "Fig. 2".

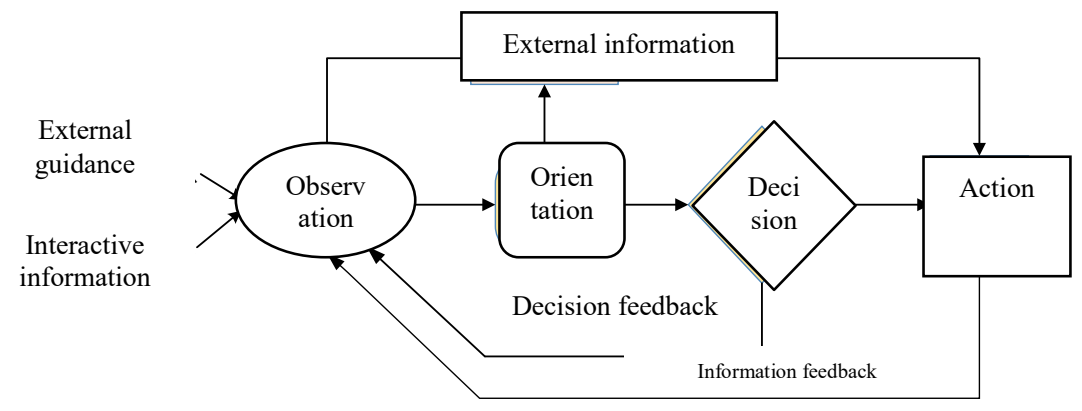

Fig. 2. OODA cycle process.

Through the analysis of the OODA ring process, we can find that the process of monitoring and early warning, judgment and positioning, plan generation and emergency response in the emergency response management of colleges and universities constitutes the four basic elements in the OODA ring.

\section{B. New Mode of Campus Emergency Management in OODA Ring}

Based on the OODA cycle theory, campus emergency management ("Fig. 3") can describe the observation process as event collection and perceiving various situations, including active perceiving monitoring video, sensors (smoke alarms), colleges and universities security offices, students, etc. And in the monitoring, it can carry out specific event collection, including canteen monitoring, dormitory monitoring, personnel monitoring and so on. Event collection runs through the life cycle of the entire campus emergency management system. The tracking and collection of comprehensive, realtime and detailed information provides strong data support for the follow-up event guidance, decision making and correction. The orientation process is abstracted by analyzing the relevant information of the collected events - the cause, the occurrence process, and the associated influences. Abstract the decision process as an event decision. The core of decisionmaking under campus emergency management is the plan, which is the specific arrangements made in advance for the staffing, facility materials, technical equipment and command actions of various departments, so as to ensure the orderly and effective handling of incidents and plan in advance. All events that have occurred and the processing of the events are samples to make the plan. Through the event evaluation of the previous step, the event collection is completed. And in this step, the selection and calling of the relevant plan are carried out. Abstract the action process as event handling, that is, the execution of the plan. Each management department realizes information sharing and complementary functions through collaborative linkage, effectively improving the effectiveness of event handling. 


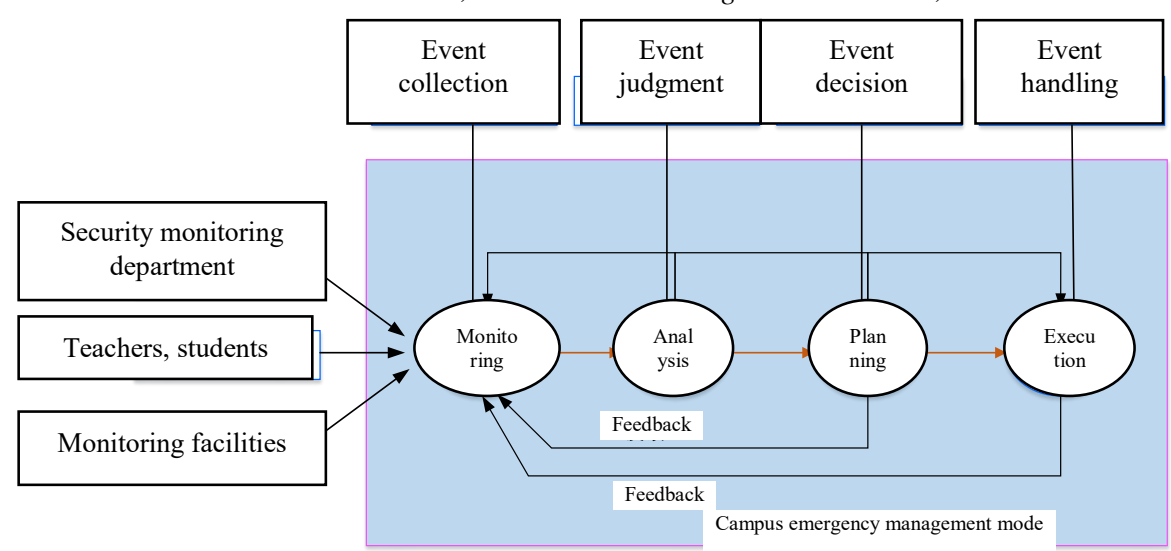

Fig. 3. Campus emergency management mode.

\section{Evaluation Index System of Campus Emergency Management Capacity}

According to the characteristics of emergency management in colleges and universities, the OODA ring theory is introduced to carry out emergency treatment from the four processes of monitoring, analysis, pre-planning and execution of emergencies. The method can effectively prevent, alert, coordinate and control emergencies, and mainly focuses on the whole process and all-round of emergency management. According to the principles on four processes of monitoring, analysis, pre-planning, and execution, and selecting scientificity, qualitative and quantitative combination, availability and dynamics in evaluation index, we can establish an evaluation index system for campus emergency management capacities for crisis monitoring and forecasting, event evaluation, crisis planning, and event processing and execution. Crisis monitoring and forecasting: emergency information platform construction, safety monitoring system, personnel safety alarm awareness, risk analysis and hidden danger investigation, early warning system; event evaluation: professional personnel quality, information analysis, information release, information research and judgment; crisis planning: emergency infrastructure, emergency technical equipment, emergency command, emergency team; event processing and execution: emergency department linkage, emergency organization, emergency control, emergency coordination. The evaluation index system for campus emergency management capacity is divided into two levels, with a total of 17 indexes, as shown in "Fig. 4".

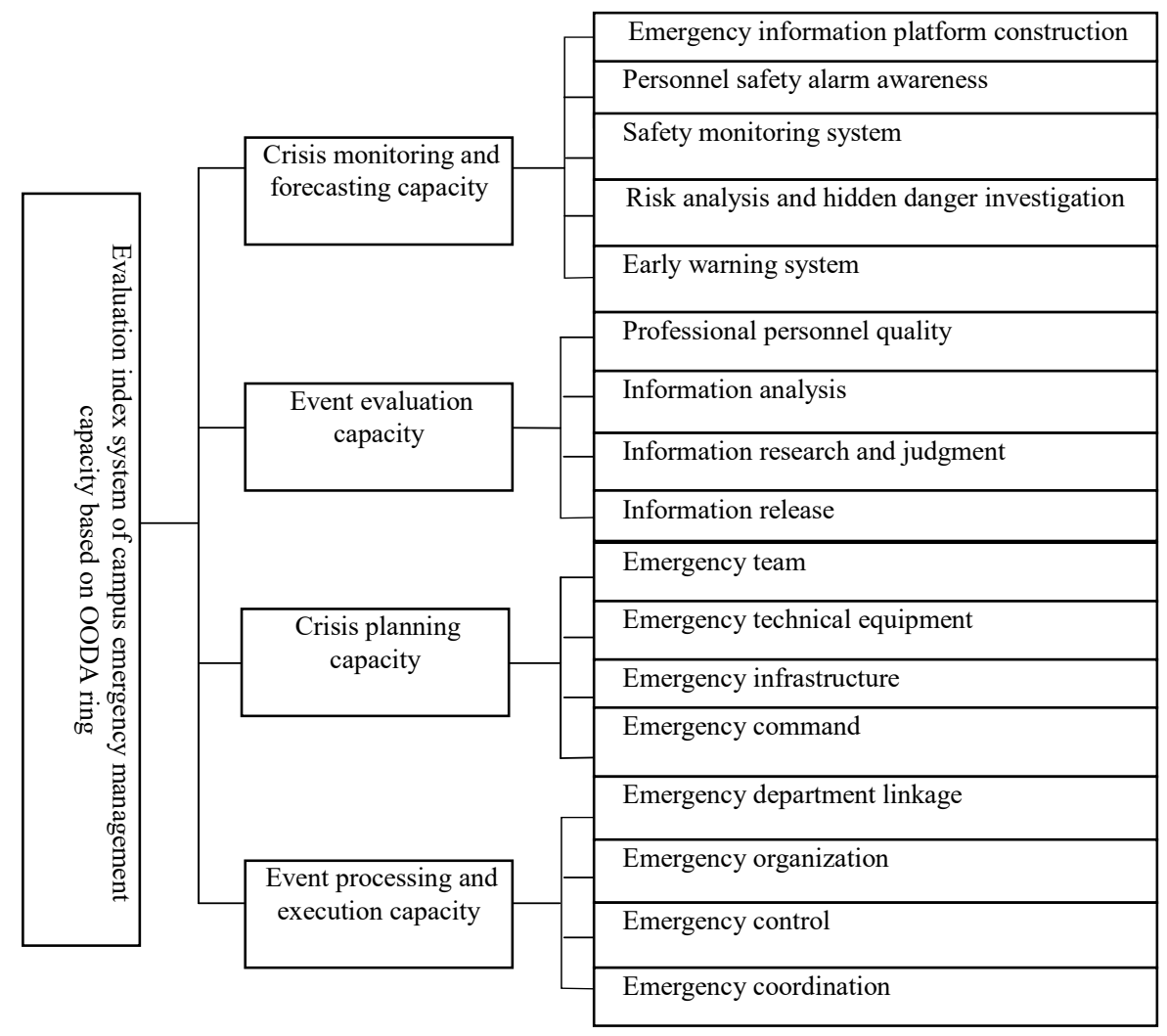

Fig. 4. Evaluation index system of campus emergency management capacity. 


\section{CONCLUSION}

Through the analysis of the concept, definition and classification of emergency management in colleges and universities, combing the research dimension of campus emergency management, clarifying the characteristics of emergency management in campus emergencies, introducing OODA ring theory and establishing a new mode of emergency management for campus emergencies. The principles of scientificity, qualitative and quantitative integration, availability and dynamics are selected, and its evaluation index system is built based on the evaluation index of emergency management capacities. Crisis monitoring and forecasting: emergency information platform construction, safety monitoring system, personnel safety alarm awareness, risk analysis and hidden danger investigation, early warning system; event evaluation: professional personnel quality, information analysis, information release, information research and judgment; crisis planning: emergency infrastructure, emergency technical equipment, emergency command, emergency team; event processing and execution: emergency department linkage, emergency organization, emergency control, emergency coordination.

\section{REFERENCES}

[1] Yan Xuxian, Dong Yan. A Review of Domestic Research Literature on Emergency Management Evaluation[J]. Journal of Business Economics, 2013(36): 102-104. (in Chinese)

[2] Chen Xinping. Research on Evaluation Index System of Community Emergency Response Capacity[J]. China Management Information Technology, 2018(7). (in Chinese)

[3] Yang Bin, Xiong Wanling, Du Fangfang. Research on the Construction of Evaluation Index System for the Whole Process of Emergency Management in Colleges and Universities[J]. China Safety Production Science and Technology, 2010, 06(4): 39-44. (in Chinese)

[4] Zha Guoqing, Xu Yani. Emergency Response Mechanism of CampusEmergencies Based on Crisis Life Cycle Theory[J]. Security, 2018(5). (in Chinese)

[5] Ruan Xingdao. Research on Safety Education Model of Colleges and Universities Based on Hall Three-Dimensional Structure[J]. Security, 2018(5). (in Chinese)

[6] Zhang Qingguo, Xu Xuejie. Analysis of Fire Safety of Campus Students Apartments by Using Safety Theory[J]. Journal of Logistics Research, 2009(4): 15-16. (in Chinese)

[7] Liu Ligong, Zhang Liping, Xue Huilai. Research on Security and Stability of Colleges and Universities Based on Analytic Hierarchy Process[J]. Journal of Hebei University of Science and Technology(Social Science Edition), 2017, 17(2): 41-46. (in Chinese)

[8] Ge Yue, Wang Tong, Xu Yabo. Evaluation of Emergency Management Capacity Based on Grey Analytic Hierarchy Process[J]. China Safety Science and Technology, 2014(12):80-86. (in Chinese)

[9] Yu Jianxin, Liu Huanchun, Wang Wenjing, et al. Campus Safety Evaluation Model and Its Application Based on BP Neural Network [J]. Safety and Environmental Engineering, 2011, 18(2): 93-95. (in Chinese)

[10] Wang $\mathrm{Xu}$, Huang Yanyan. Modeling of Urban Waterlogging Disaster Emergency Response Linkage System Based on OODA Ring[J]. Journal of Nanjing University of Science and Technology(Natural Science), 2018, 42(02). (in Chinese)

[11] Sun Rui. Research on Campus EmergenciesResponse CapacityEvaluation[D]. 2016. (in Chinese)

[12] Zhang Xu. Research on the Construction of Evaluation Index System of Emergency Management Capacity in Colleges and Universities[J]. Inner
Mongolia Science and Technology and Economy, 2015(7):23-24. (in Chinese)

[13] Lin Xian. Urban Operation Management System Based on OODA Cycle Theory[J]. Journal of Fuzhou University(Philosophy and Social Sciences), 2018(3). (in Chinese) 